\title{
Impaired Cerebroside Sulfate Hydrolysis in Fibroblasts of Sibs with "Pseudo" Arylsulfatase A Deficiency without Metachromatic Leukodystrophy
}

\author{
STEFAN J. HREIDARSSON, GEORGE H. THOMAS, ${ }^{(27)}$ HAYATO KIHARA, ARVAN L. FLUHARTY, \\ EDWIN H. KOLODNY, HUGO W. MOSER, AND LINDA W. REYNOLDS

\begin{abstract}
The John F. Kennedy Institute, Baltimore, Maryland [S.J.H., G.H.T., H.W.M., L.W.R.]; Department of Pediatrics, The Johns Hopkins University, School of Medicine, Baltimore, Maryland [S.J.H.]; Departments of Pediatrics and Medicine, The Johns Hopkins University, School of Medicine, Baltimore, Maryland [G.H.T.]; UCLA School of Medicine, MRRC-Lanterman State Hospital Research Group, Pomona, California [H.K., A.L.F.]; Eunice Kennedy Shriver Center, Waltham, Massachusetts [E.H.K.]; Departments of Neurology and Pediatrics, The Johns Hopkins University, School of Medicine, Baltimore, Maryland, USA [H.M.]
\end{abstract}

\section{Summary}

Low arylsulfatase $A$ levels are reported in two siblings, one with a neurologic disability not typical for metachromatic leukodystrophy, the other a healthy 18-year-old female with a normal developmental history. In both individuals, arylsulfatase $A$ levels in white blood cells were $7-8 \%$ of control values. Cultured fibroblasts gave low values (8-10\% of normal) for both cerebroside sulfatase and arylsulfatase $A$ activities. Other family members had enzyme levels consistent with heterozygote or normal status. Cerebroside sulfate loading tests of cultured fibroblasts in $199-\mathrm{CO}_{2}$ media were normal for all family members who were tested. In MEM-HEPES media, however, cells from the two arylsulfatase $A$ deficient siblings showed attenuated sulfolipid catabolism.

Additional clinical and laboratory studies on these individuals failed to demonstrate any features suggestive of metachromatic leukodystrophy, i.e., normal nerve conduction velocities, normal sural nerve biopsy results, and normal urinary sulfatide excretion. It is concluded that the neurologic abnormalities in the one sibling are not the result of the low enzyme activity and that both individuals represent examples of pseudo arylsulfatase $A$ deficiency (arylsulfatase A deficiency without metachromatic leukodystrophy). These results thus call into question the ability of the high-sensitivity cerebroside sulfate loading test as carried out in MEM-HEPES media to differentiate pathologically significant defects $i . e$, metachromatic leukodystrophy from benign "pseudodeficiencies."

\section{Abbreviations}

MEM-HEPES, minimum essential medium- $\mathbf{N}^{\prime}$-2-hydroxy-ethylpiperazine-N'-ethanesulfonic acid MLD, metachromatic leukodystrophy

$\mathrm{pd} / \mathrm{mld}$, pseudodeficiency/metachromatic leukodystrophy compound heterozygote

In 1963 Austin et al. (2) demonstrated that patients with metachromatic leukodystrophy lacked arylsulfatase A. Subsequently, Mehl and Jatzkewitz (16) showed that arylsulfatase A was the heat labile component of cerebroside sulfatase. In most individuals lacking this enzyme, cerebroside sulfate cleavage is blocked, cerebroside sulfate accumulates and the clinical picture of metachromatic leukodystrophy evolves over a period of time (17). Since these early observations, many investigators have provided evidence for biochemical, clinical and/or genetic heterogeneity in various subjects lacking arylsulfatase $A$; thus, metachromatic leukodystrophy can currently be subdivided into at least three distinct types, i.e., late infantile, juvenile and adult $(9,17)$. Furthermore, there have been several reports of individuals having very low arylsulfatase $\mathbf{A}$ levels but who nevertheless lack clinical features characteristic of metachromatic leukodystrophy $(4,5,6,7,11,14$, 25). Kihara et al. (11) have coined the term "pseudo arylsulfatase A deficiency" to describe these subjects whereas others have prefered the designation "arylsulfatase A deficiency without metachromatic leukodystrophy." Current data indicate that this is a benign variant having no direct medical consequences (9).

Diagnostic problems do arise, however, as arylsulfatase $A$ and cerebroside sulfatase levels are very low in both the various forms of MLD and "pseudo" arylsulfatase A deficiency. It is often difficult, on enzymatic grounds, to distinguish between these various possibilities. As a result of this problem, Fluharty et al. (7, 11) developed a cerebroside sulfate loading test that has proved useful in differentiating fibroblasts of "pseudo" deficient subjects from those of patients affected with MLD. It has recently been recognized, however, that the sensitivity of the cerebroside sulfate loading test can be influenced by variations in culture media. For example, HEPES-containing media provides slower cerebroside sulfate clearance (12). Nonetheless, in most instances studied to date, loading in MEM-HEPES has provided the most accurate reflection of the presence or absence of the clinical manifestations of MLD in individuals from whom the cells were derived.

We herein present clinical and biochemical information on a family where two individuals have low levels of arylsulfatase $A$ in leukocytes and cultured fibroblasts. One of them does show a substantial developmental disability and retardation, but there is no indication of metachromatic leukodystrophy in either individual. Yet cerebroside sulfate loading of fibroblasts from these individuals showed impaired cerebroside sulfate catabolism in MEM-HEPES media, with the cells from the neurologically normal individual showing the most profound deficit. The most satisfactory explanations for the present results are that these sisters have "pseudo" arylsulfatase A deficiency without metachromatic leukodystrophy. The neurologic impairment noted in the neurologically normal sib is unrelated and the cerebroside sulfate loading of fibroblasts cultured in MEM-HEPES media does not always provide an accurate reflection of the clinical situation.

\section{SUBJECTS}

Subject II-5 (see Fig. 1) is the product of an unremarkable pregnancy and delivery to a 39-year-old mother and a 42-year-old father. Growth and development were reported to have been normal for the first 6 months of life. At that time, slowing and 


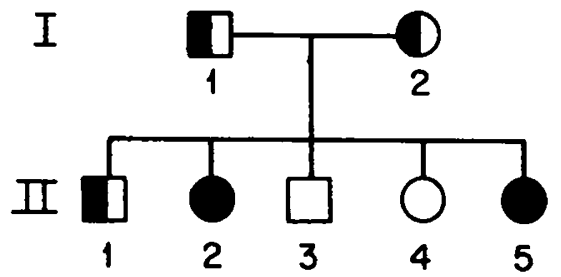

Fig. 1. Family pedigree. Dark shading represents any gene for low arylsulfatase A activity. II-2 and II-5 may be compound heterozygotes.

Table 1. Arylsulfatase A, cerebroside sulfatase activity, and urinary sulfatides

\begin{tabular}{|c|c|c|c|c|c|}
\hline \multirow[b]{2}{*}{ Individual } & \multirow[b]{2}{*}{$\begin{array}{l}\text { Pedi- } \\
\text { gree no. }\end{array}$} & \multicolumn{2}{|c|}{ Fibroblasts } & \multirow{2}{*}{$\begin{array}{l}\text { White } \\
\text { Blood } \\
\text { Cells } \\
\text { Arylsul- } \\
\text { fatase } \\
\mathbf{A}^{\mathbf{2}}\end{array}$} & \multirow[b]{2}{*}{$\begin{array}{l}\text { Urine Sul- } \\
\text { fatides }^{3}\end{array}$} \\
\hline & & $\begin{array}{l}\text { Arylsul- } \\
\text { fatase } A^{2}\end{array}$ & $\begin{array}{l}\text { C. sul- } \\
\text { fatase' }\end{array}$ & & \\
\hline \multicolumn{6}{|l|}{ Family } \\
\hline Father & $1-1$ & 237 & 26 & 31 & 0.12 \\
\hline Mother & $1-2$ & 180 & 35 & 21 & 0.08 \\
\hline Son & $11-1$ & 169 & 29 & 26 & 0.07 \\
\hline Daughter & $11-2$ & 36 & 8 & 7 & 0.06 \\
\hline Son & $11-3$ & 291 & 75 & 57 & 0.02 \\
\hline Daughter & $11-4$ & 276 & 51 & 51 & 0.15 \\
\hline Daughter & $11-5$ & 27 & 7 & 6 & 0.22 \\
\hline \multicolumn{6}{|l|}{ MLD Patients } \\
\hline Average & & 12 & 1.6 & 10 & 3.2 \\
\hline Range & & $6-16$ & $0-4$ & $5-17$ & $1.6-4.8$ \\
\hline \multicolumn{6}{|l|}{ Controls } \\
\hline Average & & 354 & 81 & 85 & 0.12 \\
\hline Range & & $278-474$ & $50-104$ & $60-119$ & $0.02-0.23$ \\
\hline
\end{tabular}

\footnotetext{
${ }^{1}$ nmole of $\left[{ }^{3} \mathrm{H}\right]$ cerebroside sulfate cleaved per $\mathrm{mg}$ protien per $\mathrm{h}$.

${ }^{2}$ nmole of $p$-nitrocatechol sulfate cleaved per $\mathrm{mg}$ protein per $\mathrm{h}$.

${ }^{3}$ nmole of sulfatide per $\mathrm{mg}$ of creatinine.
}

subsequent regression in developmental milestones were noted. A decreased rate of head growth was also documented. After 2 years of age, she began slowly to gain additional developmental and motor milestones. Examinations at $2 \frac{1}{2}$ years of age revealed microcephaly, hypotonia, and psychomotor retardation. Her EEG was mildly abnormal with occasional seizure discharges. Extensive evaluation failed to reveal any infective, metabolic, neoplastic, or vascular causes for her disorder. Subject II-2 (see Fig. 1) is an 18year-old healthy female of at least normal intelligence and athletic ability. The remaining members of the immediate family are healthy and of at least normal intelligence.

\section{MATERIALS AND METHODS}

Fibroblast cultures were established from skin biopsies obtained from the five sibs, their parents, and controls as previously described $(13,23,24)$. Arylsulfatase $A$ activity in fibroblasts was measured with 4-nitrocatechol sulfate according to a modification (24) of the method of Baum et al. (3). Cerebroside sulfatase activity was assayed with $\left.{ }^{3} \mathrm{H}\right]$ cerebroside sulfate by the method of Raghaven et al. (22). Urinary sulfatide levels were measured by high performance liquid chromatography of benzoyl derivatives as described by Nonaka and Kishimoto (18).

Cerebroside sulfate loading tests were carried out by incubating cells with $\left[{ }^{36} \mathrm{~S}\right]$-labeled rat brain cerebroside sulfate as described by Kihara et al. (11). Loading experiments designated " $199-\mathrm{CO}_{2}$ " were carried out in medium 199 with Earles salts, $26 \mathrm{mM} \mathrm{NaHCO}_{3}$ and $10 \%$ serum in an atmosphere of $5 \% \mathrm{CO}_{2}-95 \%$ air (11). Experiments labeled "MEM-HEPES" were performed on cells incubated in MEM-HEPES as described by Fluharty et al. (8).

\section{RESULTS}

Sonicates of cultured fibroblasts from II-2 and II-5 had arylsulfatase $A$ levels that were 10 and $8 \%$, respectively, of control values (Table 1). Cells from sib II-1 and the parents (I-1 and I-2) contained levels that were 67,51 and $48 \%$, respectively, of normal. Arylsulfatase A activities of cell sonicates of sibs II-3 and II-4 were in the range of control cells (Table 1). Similar, but somewhat lower, results were obtained with leukocyte preparations (Table 1).

Cerebroside sulfatase activities in fibroblast sonicates of these individuals against $\left[{ }^{3} \mathrm{H}\right.$ ]-labeled cerebroside sulfate are summarized in Table 1. Again, cells from subjects II-2 and II-5 had low levels whereas sib II-1 and the parents had intermediate levels.

The ability of subjects I-1, I-2, II-2, and II-5 to metabolize cerebroside sulfate was also examined by culturing intact fibroblasts in media containing $\left[{ }^{35} \mathrm{~S}\right]$-labeled substrate for 2,4 , and 6 days. These loading tests were carried out in two media: in the usual $199-\mathrm{CO}_{2}$ and in a MEM-HEPES system, which tends to be more sensitive to marginal cerebroside sulfatase function. In the 199- $\mathrm{CO}_{2}$ system, fibroblasts from both II-2 and II-5 degraded the labeled sulfolipid in a nearly normal manner (Fig. 2). In MEMHEPES, a distinct impairment in the ability to degrade cerebroside sulfate was evident in both enzyme deficient cell lines (Fig. 3). The impairment was most severe in cells from the normal subject II-2, falling into a range more or less typical of juvenile or adult MLD cells in this medium. The defect in the neurologically impaired subject II-5 was less striking and was in the range encountered with pseudo-deficiency cell lines. Fibroblasts from I1 and I-2 were indistinguishable from controls in both media.

\section{DISCUSSION}

These studies demonstrate that subjects II-2 and II-5 have low levels of both arylsulfatase $A$ and cerebroside sulfatase activities; moreover, intermediate levels of these activities in the cells of the parents and one sib indicate that the alterations are inherited in an autosomal recessive manner. Additional investigations of subjects II-2 and II-5 failed to provide any evidence indicating that these enzyme deficiencies had any direct clinical consequences. Although patient II-5 suffers from psychomotor retardation, microcephaly, and hypotonia, she nevertheless lacks any clinical or biochemical abnormalities compatible with any form of metachromatic leukodystrophy. Specifically, the following procedures

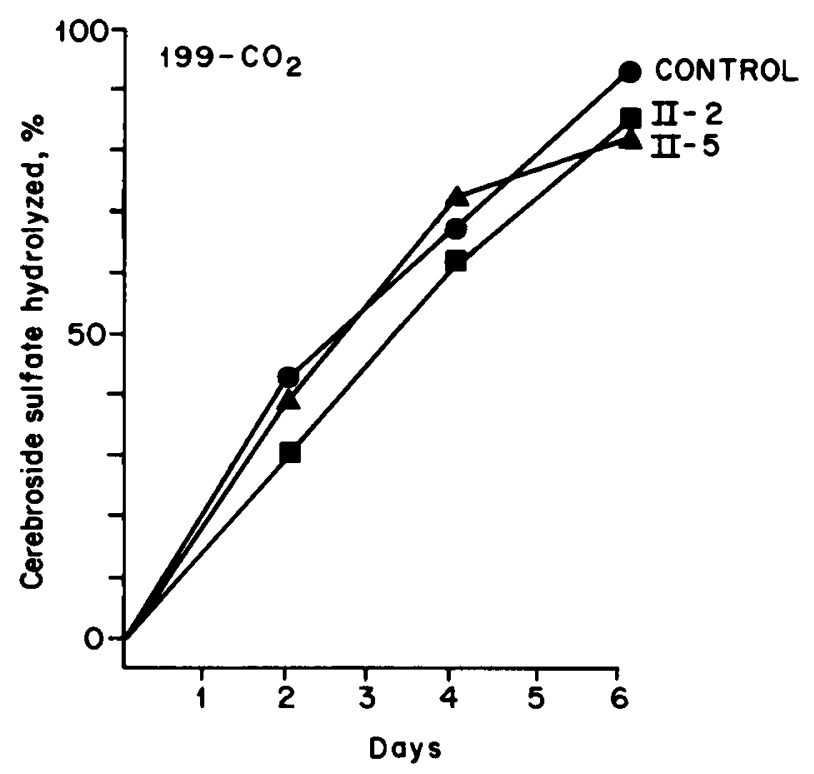

Fig. 2. Cerebroside sulfate loading test of low enzyme fibroblast cultures in 199- $\mathrm{CO}_{2}$ media. $\mathrm{O}$, normal; $\Delta$, patient II-5; and 2 patient II-2. 


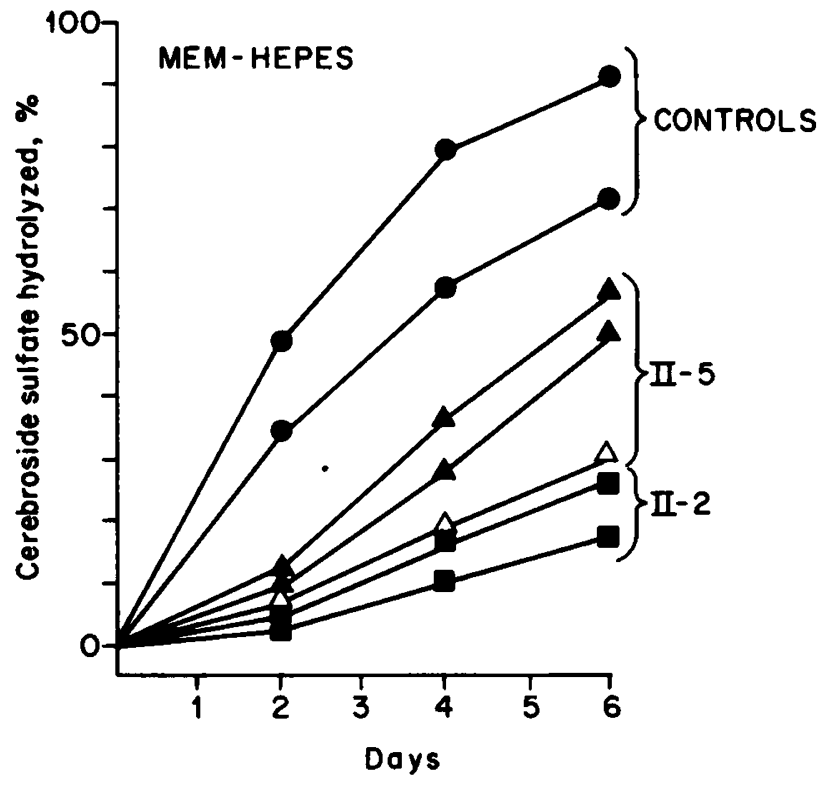

Fig. 3. Cerebroside sulfate loading test of low enzyme fibroblast cultures in MEM-HEPES media. The results of two separate experiments are presented with the same control cell line run on both occasions. This is typical of the range of normal controls in this medium. , normal control; $\Delta \Delta$, patient Il-5 with open and solid symbols representing separate sublines; and $\mathrm{L}$ patient II-2.

yielded normal results: measurement of nerve conduction velocities, analysis of cerebrospinal fluid protein, ophthalmologic examination, computed cerebral tomography and brainstem auditory evoked responses. In addition, microscopy of plastic-embedded sections of a sural nerve biopsy which were stained with toluidine blue, showed no evidence of demyelination, axonal loss, or cell inclusion.

Normal levels of sulfatides in the urine of both II-2 and II-5 (see Table 1) also provide direct evidence that these individuals, in contrast to MLD patient (19), are capable of metabolizing cerebroside sulfates in a normal fashion. The normal development of subject II-2 also supports this conclusion.

We can not rule out the possibility of an adult or a variant form of metachromatic leukodystrophy in the preclinical stage existing in either of these sibs; however, the results of the additional laboratory procedures make this a very unlikely possibility. Preclinical forms of adult metachromatic leukodystrophy would be expected to have increased levels of urinary sulfatides (20), abnormal sural nerve biopsy results (1), and decreased nerve conduction times (20), all of which are normal in these individuals.

Until now the sulfatide loading test has been an accurate means of distinguishing between "pseudo" deficiency and the various clinical forms of MLD. The sensitivity of the test to marginal cerebroside sulfatase function is enhanced when MEM-HEPES rather than the original $199 \cdot \mathrm{CO}_{2}$ system (21) is employed (12). Normal individuals with low arylsulfatase $A$ activities have, heretofore, been nearly indistinguishable from controls in either growth media. A diminution of cerebroside sulfate clearance capacity in "pseudo"-deficient cell lines has been seen in MEMHEPES, but clearance of less than half the assimilated sulfolipid has only been encountered in neurologically impaired individuals. Even though subjects II-5 might be included in this category, subject II-2 cannot. A clear depression of cerebroside sulfate catabolism in cells cultured MEM-HEPES occurs without any indication of neurologic compromise or aberrant in vivo sulfolipid metabolism in the intact individual. Furthermore, there appears to be no association between low arylsulfatase $A$ activity, the moderate cerebroside sulfate clearance defect in MEM-HEPES and the "non-MLD" neurologic abnormality in subject II-5.
We thus conclude that these two subjects can be best classified as having some form of the so called "pseudo" arylsulfatase A deficiency or "arylsulfatase A deficiency without metachromatic leukodystrophy" variant. In most cases of this type, a pd/mld genotype is presumed because MLD occurred in other family members. The two siblings in the present study could represent either $\mathrm{pd} / \mathrm{pd}$ or $\mathrm{pd} / \mathrm{mld}$ genotype because there is no evidence for MLD in the family.

A review of the medical literature indicates that the enzyme results of our cases closely parallel those of several other enzyme deficient individuals who also lack the clinical features of MLD $(4,5,8,9,11,14,22,25)$. As in our two cases, the leukocyte arylsulfatase $A$ levels have been very close to or just above those of MLD patients, whereas both arylsulfatase $A$ and cerebroside sulfatase levels in the fibroblasts have usually been just above those of MLD patients. Although the latter finding may provide an explanation for the lack of clinical disease in the "pseudo"deficient subjects, the values are often so close that an additional procedure may be desirable in an individual patient. Inasmuch as, cerebroside sulfate loading of cultured fibroblast remains a most valuable adjunct, the present results suggest that it must be interpreted with caution when clinical evidence for sulfatide accumulation is lacking. The present results thus raise some concern about the use of the cerebroside sulfate loading test for prenatal diagnoses in "pseudo"-deficient families. In all such diagnoses to date, the issue has been differentiating between late infantile MLD and "pseudo"-deficient phenotypes. This is easily done by the loading test carried out in the $199-\mathrm{CO}_{2}$ medium. In conditions where a loading defect is only manifest in the MEM-HEPES system, an accurate prenatal diagnosis could be extremely difficult if the family carried the "pseudo"-deficient trait as well.

It is notewoirthy that culture conditions have previously been shown to alter the levels of a number of sulfatases in multiple sulfatase deficiency fibroblasts (8). This disorder is excluded in our subjects by the following findings: normal arylsulfatase $B$ levels, normal [ ${ }^{35} \mathrm{SO}_{4}$ ] incorporation kinetics, normal urinary mucopolysaccharide levels, and incompatible clinical status.

Although this basis of the findings reported here remains unclear, they may provide additional clues as to the biologic alterations responsible for both the various forms of MLD as well as the "pseudo"-deficient state. Furthermore, they strongly indicate the need for carefull consideration of culture conditions when utilizing the cerebroside sulfate loading procedure for either diagnostic or investigative studies. The occurrence of severe neurologic problems in an apparently "pseudo"-deficient individual does call the benign nature of the pseudodeficiency state into question. At present this must be regarded as a fortuitous association, but if additional examples are found in the future, one would have to consider the possibility of some sort of increased risk for such individuals.

\section{REFERENCES AND NOTES}

1. Argyrakis, A., Pilz, H., Goebel, H. H., and Muller, D.: Ultrastructural findings of peripheral nerve in a preclinical case of adult metachromatic leukodystrophy. J. Neuropath. Exp. Neurol., 36: 693 (1977).

2. Austin, J. H., Balasubramanian, A. S., Pattabiraman, T. N., Saraswathi, S., Basu, D. K., and Bachhawat, B.: A controlled study of enzymic activities in three humnen disorders of glycolipid metabolism. J. Neurochem., 10: 805 (1963).

3. Baum, H., Dodfon, K. S., and Spencer, B.: The assay of arylsulfatase $A$ and $B$ in human urine. Clin. Chim. Acta, 4: 453 (1959).

4. Butterworth, J., Broedhead, D. M., and Keay, A. J.: Low arylsulphatase A activity in a family without metachromatic leukodystrophy. Clin. Genet., 14: 213 (1978).

5. Dubois, G., Tuppin, J., and Baumann, N.: Absence of ASA activity in healthy father of a patient with metachromatic leukodystrophy. N. Engl. J. Med., 293: 302 (1975).

6. Dubois, G., Harzer, K., and Baumann, N.: Very low arylsulfatase $A$ and cerebroside sulfatase activities in leukocytes of healthy members of metachromatic leukodystrophy family. Am. J. Hum. Genet., 29: 191 (1977).

7. Fluharty, A. L., Stevens, R. L., and Kihara, H.: Cerebroside sulfate hydrolysis by fibroblasts from a metachromatic leukodystrophy parent with deficient arylsulfatase A. J. Pediatr., 92: 782 (1978).

8. Fluharty, A. L., Stevens, R. L. de la Flor, S. D., Shapiro, L. J., and Kihara, H.: 
Arylsulfatase A modulation with $\mathrm{pH}$ in multiple sulfatase deficiency disorder fibroblasts. Am. J. Hum. Genet., 31: 574 (1979).

9. Kihara, H.: Genetic heterogeneity in metachromatic leukodystrophy. Am. J. Hum. Genet., 34: 171 (1982).

10. Kihara, H., Fluharty, A. L., O'Brien, J. S., and Fish, C. H.: Metachromatic leukodystrophy caused by a partial cerebroside sulfatase defect. Clin. Gen., 21: 253 (1982).

11. Kihara, H., Ho, C., Fluharty, A. L., Tsay, K. K., and Hartlage, P. L.: Prenatal diagnosis of metachromatic leukodystrophy in a family with pseudo arylsulfatase A deficiency by the cerebroside sulfate loading test. Pediatr. Res., 14: 224 (1980).

12. Kihara, H., Tsay, K. K., and Fluharty, A. L.: Effect of HEPES on the fibroblast cerebroside sulfate loading test. Biochem. Med., (In press).

13. Lie, K. K., Thomas, G. H. Taylor, H. A., and Sensenbrenner, J. A.: Analysis of $\mathrm{N}$-acetyl- $\beta$-D-glucosaminidase in mucolipidosis II (I-cell disease). Clin. Chim. Acta, 45: 243 (1973).

14. Lott, I. T., Dulaney, J. T., Milunsky, A., Hoefnagel, D., and Moser, H. W.: Apparent biochemical homozygosity in two obligatory heterozygotes for metachromatic leukodystrophy. J. Pediatr., 89: 438 (1976).

15. Lott, I. T. and Dulaney, J. T.: Sulfatide excretion in metachromatic leukodystrophy. Am. J. Hum. Genet., 30: 228 (1978).

16. Mehl, E. and Jatzkewitz, H.: Cerebroside 3-sulfate as a physiological substrate of arylsulfatase A. Biochim. Biophys. Acta, 151: 619 (1968).

17. Moser, H. W. and Dulaney, J. T.: Sulfatide lipidosis: Metachromatic leukodystrophy. In Stanbury, J. B., Wyngaarden, J. B. and Frederickson, D. S., eds: The metabolic basis of inherited diseases, 4th ed., pp. 770-809. (McGraw-Hill, New York, 1978).

18. Nonaka, G. and Kishimoto, Y.: Simultaneous determination of picomole levels of gluco- and galactocerebroside, monogalactosyl diglyceride, and sulfatide by high performance liquid chromatography. Biochim. Biophys. Acta, 572: 423 (1979).
19. Philippart, M., Sarlieve, L., Meurant, C., and Mechler, L.: Human urinary sulfatides in patients with sulfatidosis (metachromatic leukodystrophy). J. Lipid Res., 12: 434 (1971).

20. Pilz, H. and Hopf, H. C.: A preclinical case of late adult metachromatic leukodystrophy. J. Neurol. Neurosurg. Psychiatry, 35: 360 (1972).

21. Porter, M. T., Fluharty, A. L., Trammel, J., and Kihara, H.: A correlation of intracellular cerebroside sulfatase activity in fibroblasts with latency in metachromatic leukodystrophy. Biochem. Biophys. Res. Commun., 44: 660 (1971)

22. Raghavan, S. S., Gajewski, A., and Kolodny, E. H.: Leukocyte sulfatidase for the reliable diagnosis of metachromatic leukodystrophy. J. Neurochem., 36: 724 (1981).

23. Taylor, H. A., Thomas, G. H., Miller, C. S., Kelly, T. E., and Siggens, D.: Mucolipidosis III (pseudo-Hurler polydystrophy): cytological and ultrastructural observations of cultured fibroblast cells. Clin. Genet., 4: 388 (1973).

24. Thomas, G. H., Taylor, H. A., Reynolds, L. W., and Miller. C. S.: Mucolipidosis III (pseudo-Hurler polydystrophy): Multiple lysosomal enzyme abnormalities in serum and cultured fibroblast cells. Pedintr. Res., 7: 751 (1973).

25. Weiter, J. J., Feingold, M., Kolodny, E. H., and Raghavan, S. S.: Retinal pigment epithelial degeneration associated with leukocytic arylsulfatase $A$ deficiency. Am. J. Ophthalmol., 90: 768 (1980).

26. The authors thank Dr. Srinivasa Raghavan for his kind gift of $\left[{ }^{3} \mathrm{H}\right]$ labeled cerebroside sulfate utilized for the determination of fibroblast sulfatidase activity.

27. Requests for reprints should be addressed to: George H. Thomas, Ph.D., The John F. Kennedy Institute, 707 N. Broadway, Baltimore, MD 21205 (USA).

28. This study was supported by Program Project Grants No. 917 from Maternal and Child Health, Dept. of Human Services and Grant No. HD 10981-05, NS11665 and HD 4612 . National Institutes of Health.

29. Received for publication July $27,1982$.

30. Accepted for publication December 21, 1982.

\title{
Selective Fetal Malnutrition: the Effect of in Vivo Ethanol Exposure upon in Vitro Placental Uptake of Amino Acids in the Non-Human Primate
}

\author{
STANLEY E. FISHER, ${ }^{(29)}$ MARK ATKINSON, STANLEY JACOBSON, PREBHAT SEHGAL, \\ JOHN BURNAP, EDWARD HOLMES, SAUL TEICHBERG, ELLEN KAHN, RONALD JAFFE, AND \\ DAVID H. VANTHIEL

\begin{abstract}
Department of Pediatrics, North Shore University Hospital and Cornell University Medical College, Manhasset, New York, USA [S.E.F., M.A., S.T.]; Department of Anatomy and Cellular Biology, Tufts University School of Medicine, Boston, Massachusetts, USA [S.J., J.B., E.H.,]; Department of Primate Medicine, New England Regional Primate Research Center and Harvard Medical School, Southborough, Massachusetts, USA [P.S.]; Department of Pathology and Laboratories, North Shore University Hospital and Cornell University Medical College, Manhasset, New York, USA [E.K.]; Department of Pathology, University of Pittsburgh School of Medicine, Pittsburgh, Pennsylvania, USA [R.J.]; and Department of Medicine, University of Pittsburgh School of Medicine, Pittsburgh, Pennsylvania, USA [D.H.V.T.]
\end{abstract}

\section{Summary}

In vitro uptake (45 and 90 minutes) of amino acids, alpha-amino isobutyric (AIB) and valine (VAL), was measured in six placentae from the nonhuman primate, Macacca fascicularis. Three of the pregnant primates were chronically treated with ethanol before and throughout pregnancy (CHR); one during the last trimester only (LT); and two were controls (C). Compared to the C placentae, the LT placenta had significantly decreased uptake only for AIB at 45 min: $33.4 \pm 6.8 \%$ reduction (mean \pm S.E.) $(P<0.05)$. In contrast, the CHR placentae demonstrated significantly reduced uptake $(P<0.01)$ for both amino acids at both time points. Percent reduction at 45 and 90 min: AIB, $35.2 \pm 6.5 \%$ and 32.6 $\pm 5.6 \%$ and VAL, $38.7 \pm 2.9 \%$ and $22.1 \pm 4.1 \%$. The results indicate that chronic in vivo ethanol exposure impairs the in vitro placental uptake of two actively transported amino acids, using an animal with a placenta almost identical to the human.

\section{Abbreviations}

AIB, alpha-amino isobutyric acid

$C_{\mathfrak{V}} / C_{0}$, ratio of intracellular to extracellular concentration

C, control

CHR, chronic

FAS, fetal alcohol syndrome

In, inulin

IUGR, intrauterine growth retardation

LT, last trimester

VAL, valine 